\title{
Urinary iodine level and its determinants in pregnant women of Shanghai, China
}

\author{
Zhenzhen Wei ${ }^{1} \dagger$, Weiye Wang ${ }^{1} \dagger$, Jun Zhang ${ }^{1}$, Xiaohua Zhang ${ }^{2}$, Longmei Jin $^{2}$ and Xiaodan Yu ${ }^{1 *} \neq$ \\ ${ }^{1}$ MOE-Shanghai Key Laboratory of Children's Environmental Health, Development of Child and Adolescent Healthcare, \\ XinHua Hospital Affiliated to Shanghai Jiao Tong University School of Medicine, Shanghai, People's Republic of China \\ ${ }^{2}$ Minhang District Maternal and Child Health Hospital, Shanghai, People's Republic of China \\ (Submitted 22 September 2014 - Final revision received 29 January 2015 - Accepted 9 February 2015 - First published online 8 April 2015)
}

\section{Abstract}

It is known that iodine deficiency during pregnancy can interfere with normal fetal growth and development. However, iodine levels of pregnant women in Shanghai, China, and factors that could influence its levels remain unclear. A total of 916 pregnant women were selected from the Maternal and Child Care Service Centre of Minhang District in Shanghai. Morning urinary iodine (UI) and iodine content of salt from the participants' home were measured, and UI concentration was adjusted by creatinine concentrations. Serum tri-iodothyronine, thyroxin, free tri-iodothyronine, free thyroxine and thyroid-stimulating hormone were tested in the second trimester of pregnancy by time-resolved fluoroimmunoassay. The median levels of UI in pregnant women were 156.3, 176.9 and $175 \cdot 1 \mu \mathrm{g} / \mathrm{g}$ creatinine in the first, second and third trimesters of pregnancy, respectively. The prevalence of UI deficiency (UI $<150 \mu \mathrm{g} / \mathrm{g}$ creatinine) was $48.3,34.2$ and $36.2 \%$ in the three trimesters of pregnancy, respectively. Factors that significantly influenced the UI levels include the following: iodine content of household salt; age; occupation; multivitamin supplement with iodine; seaweed intakes. Furthermore, UI and iodine content of salt were moderately correlated $(r 0.406, P<0.001)$. In addition, there was no significant association between UI and thyroid hormone levels. The present study showed a high prevalence of UI deficiency in pregnant women in Shanghai, especially during the first trimester of pregnancy. Both iodine content of household salt and multivitamin supplement with iodine are the main determinants of UI levels in Shanghai.

\section{Key words: Urinary iodine: Iodised salt: Influencing factors: Thyroid hormones: Pregnant women}

Iodine is an essential micronutrient needed for fetal neurodevelopment and thyroid hormone production ${ }^{(1)}$. In early pregnancy, fetal brain development depends on maternal thyroid hormone, whereas iodine deficiency leads to inadequate production of thyroid hormones ${ }^{(2)}$. A recent medical news and perspectives in 2010 has reported that an estimated 38 million babies born each year in developing countries are at risk for developing brain damage that is associated with iodine deficiency ${ }^{(3,4)}$. Furthermore, low birth weight or hearing loss in babies is also associated with prenatal iodine deficiency $^{(5,6)}$. Therefore, achieving adequate iodine nutrition during pregnancy is an important public health objective.

The key to achieving the best iodine status in the pregnant population is to determine the sources of iodine. Foods are the major sources of the required iodine in the human body, which accounts for more than $90 \%$ of iodine intake, and the largest fraction of dietary iodine is excreted by the kidneys ${ }^{(7)}$.
The excretion of iodine in the urine is a good measure of iodine intake. Urinary iodine (UI) is considered as a reliable indicator reflecting the latest iodine intake, and as a safety biomarker linking closely to iodine metabolism during pregnancy ${ }^{(4,8)}$.

China is one of the countries in which iodine deficiency is due to the deficiency of environmental sources. Since 1996, Shanghai has carried out the universal salt iodisation strategy. In the past, Shanghai was not considered an iodine-deficient area. A recent study in Shanghai has shown that the current dietary iodine intake is sufficient in the general population, but insufficient especially in pregnant women ${ }^{(9)}$. However, that study has not reported the prevalence of iodine deficiency in different trimesters of pregnancy or the sources of dietary iodine intake in pregnant women. Therefore, in the present study, we examined dietary factors that influence the iodine level during pregnancy using a questionnaire including sources of dietary iodine intake. Furthermore, we analysed

\footnotetext{
Abbreviations: T3, tri-iodothyronine; T4, thyroxin; FT3, free tri-iodothyronine; FT4, free thyroxine; TSH, thyroid-stimulating hormone; UI, urinary iodine *Corresponding author: Dr X. Yu, fax +862125078875, email xd_yu2003@126.com

† Co-first authors.

‡Present address: 1665 Kongjiang Road, Shanghai, People’s Republic of China.
} 
the association between iodine content of salt and secretion in the urine. We intended to clarify the iodine status in the three trimesters of pregnancy, the determinants of UI level, and the association between thyroid hormone and UI levels in pregnant women.

\section{Materials and methods}

\section{Subjects}

From 2011 to 2012, a total of 1102 pregnant women who lived in Shanghai for at least 3 years were recruited from the clinics of the Maternal and Child Care Service Centre of Minhang District, Shanghai for the present study. Finally, a sample of 916 pregnant women, with 211, 360 and 345 participants in the first, second and third trimesters, respectively, signed the written informed consent form. A first-morning spot urine sample was collected, and a questionnaire was conducted by face-to-face interviews.

Of the 360 participants in the second trimester, 180 were randomly selected and blood samples were collected for the measurement of serum tri-iodothyronine (T3), thyroxin (T4), free tri-iodothyronine (FT3), free thyroxine (FT4) and thyroidstimulating hormone (TSH). Of the 180 participants, 134 gave their written informed consent for blood sample collection. The present study was approved by the Ethics Committee of Xinhua Hospital, Shanghai Jiao Tong University School of Medicine.

\section{Questionnaire information}

From the questionnaire, data on socio-economic status, medical history of thyroid disease (hyperthyroidism, hypothyroidism, goitre, thyroid cyst and thyroid cancer), smoking status and sources of dietary iodine intake in the $24 \mathrm{~h}$ recall were collected. Data on the socio-economic status of each participant provided information on residence, occupation, educational levels of couples, and household incomes. Any source of dietary iodine intake in the $24 \mathrm{~h}$ recall, such as iodised salt, multivitamin supplement with iodine, cows' milk, yogurt, soya sauce, egg and aquatic foods, was collected.

\section{Measurement of urinary iodine and iodine content of salt}

The first-morning spot urine sample was collected from each participant in a clean cup and stored at $-40^{\circ} \mathrm{C}$ until urine test for iodine. Participants were asked to bring a small bottle of salt from their home. UI was determined by the modified Sandell-Kolthoff reaction, as described previously ${ }^{(10)}$, which was a standard promulgated by the Ministry of Health of the People's Republic of China (WS/T 107-2006). The urine specimen was first digested with chloric acid, and iodine was determined from its catalytic reaction of ceric ammonium sulphate in the presence of arsenious acid ${ }^{(10)}$. Concentrations of urinary creatinine were determined by an automated colorimetric method on a Beckman Synchron LX20 or CX3 clinical analyser, as described previously ${ }^{(11)}$. Iodine concentrations were adjusted by using creatinine concentrations to correct for variable water excretion rates at the time of spot urine specimen collection. UI concentrations are expressed as $\mu \mathrm{g} / \mathrm{g}$ creatinine. Considering the titration method with thiosulphate becomes more complicated because of the interference of $\mathrm{Fe}$ in salt ${ }^{(12)}$. Iodine content of salt was determined with the modified Sandell-Kolthoff reaction using an aliquot of $10 \mathrm{~g}$ salt in distilled water ${ }^{(13)}$.

\section{Serum thyroid-stimulating hormone, free tri-iodothyronine and free thyroxine}

In the second trimester of pregnancy, 134 blood samples for serum analysis were collected and stored at $-40^{\circ} \mathrm{C}$ after serum preparation. Serum T3, T4, FT3, FT4 and TSH were measured by time-resolved fluoroimmunoassay on an auto DELFIA analyser (Wallac Oy) ${ }^{(14)}$. FT4 testing in pregnancy is challenging, due to a number of related changes such as an alteration in the concentrations of albumin and thyroxinebinding globulin. During pregnancy, FT4 immunoassays are challenging because they may be sensitive to changes in a method-specific manner. Previous studies have proved that immunoassay may yield a FT4 pattern during pregnancy similar to that obtained by equilibrium dialysis isotope dilution-liquid chromatography/tandem $\mathrm{MS}^{(15)}$.

T3, T4, TSH, FT3 and FT4 are expressed as nmol/1, nmol/l, $\mathrm{mIU} / \mathrm{l}, \mathrm{pmol} / \mathrm{l}$ and $\mathrm{pmol} / \mathrm{l}$, respectively. In view of the controversial validity of certain immunoassays under conditions of altered serum binding proteins ${ }^{(16)}$, trimester-specific reference ranges were used. The second trimester-specific reference ranges for these hormones were as follows: T3 (1.62$3.72 \mathrm{nmol} / \mathrm{l})$; T4 $(88.6-213.2 \mathrm{nmol} / \mathrm{l})$; FT3 $(3.55-5.25 \mathrm{pmol} / \mathrm{l})$; FT4 (10.6-17.6 pmol/1); TSH $(0.05-4.5 \mu \mathrm{IU} / \mathrm{ml})^{(17)}$.

\section{Statistical analyses}

The distribution of UI concentrations was skewed towards the left. Thus, we performed $\log _{10}$ transformation before analysis. We first determined the prevalence of UI deficiency in pregnant women (Table 1). Then, we performed a univariate analysis to examine the influencing factors of UI (Table 2), and used multivariable analysis to estimate generalised linear models of UI (Table 3). The prevalence of normal thyroid

Table 1. Median urinary iodine (UI) concentrations and the prevalence of UI deficiency in pregnant women ( $n$ 916)

\begin{tabular}{lcccr}
\hline & First trimester & Second trimester & Third trimester & $P$ \\
\hline$n$ & 211 & 360 & 345 & \\
Median UI $(\mu \mathrm{g} / \mathrm{g}$ creatinine) & 156.3 & 176.9 & $175 \cdot 1$ & $<0.01$ \\
Ul deficiency, $<150 \mu \mathrm{g} / \mathrm{g}$ creatinine $(\%)$ & 48.3 & 34.2 & 36.2 & $<0.01$ \\
\hline
\end{tabular}


Table 2. Influencing factors of urinary iodine (UI) by the univariate analysis

(Mean values and standard deviations, $n$ 916)

\begin{tabular}{|c|c|c|c|c|}
\hline & \multicolumn{3}{|c|}{$\log _{10}$ UI $(\mu \mathrm{g} / \mathrm{g})$} & \multirow[b]{2}{*}{$P$} \\
\hline & $n$ & Mean & SD & \\
\hline \multicolumn{5}{|l|}{ Socio-economic status } \\
\hline Occupation (\%) & & & & $>0.05$ \\
\hline White collar & 433 & $2 \cdot 24$ & 0.26 & \\
\hline Blue collar & 46 & 2.34 & 0.22 & \\
\hline Housewife & 156 & $2 \cdot 26$ & 0.27 & \\
\hline Other & 260 & $2 \cdot 25$ & 0.29 & \\
\hline Educational level (\%) & & & & $>0.05$ \\
\hline Middle school or lower & 84 & $2 \cdot 28$ & 0.28 & \\
\hline High school & 165 & $2 \cdot 28$ & 0.26 & \\
\hline Some college/bachelor's degree or higher & 665 & $2 \cdot 25$ & 0.27 & \\
\hline Husband's educational level (\%) & & & & $>0.05$ \\
\hline Middle school or lower & 59 & $2 \cdot 25$ & 0.27 & \\
\hline High school & 130 & $2 \cdot 27$ & 0.25 & \\
\hline Some college/bachelor's degree or higher & 720 & $2 \cdot 25$ & 0.27 & \\
\hline Household income (yuan/month per person) & & & & $>0.05$ \\
\hline$<2000$ & 30 & $2 \cdot 31$ & 0.24 & \\
\hline $2000-5000$ & 257 & $2 \cdot 28$ & 0.27 & \\
\hline $5000-10000$ & 406 & $2 \cdot 23$ & 0.26 & \\
\hline$>10000$ & 213 & 2.25 & 0.29 & \\
\hline \multicolumn{5}{|l|}{ Source of dietary iodine intake in the $24 \mathrm{~h}$ recall } \\
\hline lodised salt used & & & & $<0.001$ \\
\hline No & 136 & 2.00 & 0.22 & \\
\hline Yes & 779 & $2 \cdot 30$ & 0.22 & \\
\hline \multicolumn{2}{|l|}{ Multivitamin with iodine $(15 \mu \mathrm{g} / \mathrm{d})$} & & & $<0.001$ \\
\hline No & 849 & $2 \cdot 24$ & 0.27 & \\
\hline Yes & 66 & 2.37 & 0.27 & \\
\hline \multicolumn{2}{|l|}{ Seaweed } & & & $<0.001$ \\
\hline No & 785 & $2 \cdot 23$ & 0.25 & \\
\hline Yes & 131 & 2.43 & 0.31 & \\
\hline \multicolumn{2}{|l|}{ Cows' milk } & & & $>0.05$ \\
\hline No & 348 & $2 \cdot 23$ & 0.29 & \\
\hline Yes & 568 & $2 \cdot 27$ & 0.26 & \\
\hline \multicolumn{2}{|l|}{ Yogurt } & & & $>0.05$ \\
\hline No & 658 & $2 \cdot 25$ & 0.27 & \\
\hline Yes & 257 & $2 \cdot 26$ & 0.26 & \\
\hline \multicolumn{2}{|l|}{ Soya sauce } & & & $>0.05$ \\
\hline No & 279 & $2 \cdot 26$ & 0.29 & \\
\hline Yes & 638 & $2 \cdot 25$ & 0.26 & \\
\hline \multicolumn{2}{|l|}{ Egg } & & & $>0.05$ \\
\hline No & 295 & $2 \cdot 23$ & 0.28 & \\
\hline Yes & 621 & $2 \cdot 26$ & 0.26 & \\
\hline \multicolumn{2}{|l|}{ River fish } & & & $>0.05$ \\
\hline No & 740 & $2 \cdot 25$ & 0.28 & \\
\hline Yes & 176 & $2 \cdot 27$ & 0.24 & \\
\hline Sea fish & & & & $>0.05$ \\
\hline No & 763 & $2 \cdot 26$ & 0.27 & \\
\hline Yes & 152 & $2 \cdot 24$ & 0.26 & \\
\hline Clams & & & & $>0.05$ \\
\hline No & 896 & $2 \cdot 25$ & 0.27 & \\
\hline Yes & 20 & $2 \cdot 30$ & 0.27 & \\
\hline Shrimp & & & & $>0.05$ \\
\hline No & 785 & $2 \cdot 26$ & 0.27 & \\
\hline Yes & 130 & $2 \cdot 24$ & 0.25 & \\
\hline Crab & & & & $>0.05$ \\
\hline No & 892 & $2 \cdot 26$ & 0.27 & \\
\hline Yes & 23 & $2 \cdot 15$ & 0.27 & \\
\hline Medical history of thyroid & & & & \\
\hline Disease of thyroid & & & & $>0.05$ \\
\hline No & 880 & $2 \cdot 26$ & 0.27 & \\
\hline Yes & 36 & 2.23 & 0.24 & \\
\hline Medicine containing iodine & & & & $>0.05$ \\
\hline No & 908 & $2 \cdot 26$ & 0.27 & \\
\hline Yes & 8 & $2 \cdot 20$ & 0.21 & \\
\hline Smoking & & & & \\
\hline Smoking during pregnancy & & & & $>0.05$ \\
\hline No & 910 & $2 \cdot 25$ & 0.27 & \\
\hline Yes & 5 & $2 \cdot 32$ & 0.27 & \\
\hline Second-hand smoking & & & & $>0.05$ \\
\hline No & 412 & $2 \cdot 26$ & 0.25 & \\
\hline Yes & 503 & $2 \cdot 25$ & 0.28 & \\
\hline
\end{tabular}


Table 3. Generalised linear models of urinary iodine (UI)* (Coefficients with their standard errors, $n$ 916)

\begin{tabular}{lccc}
\hline & \multicolumn{2}{c}{$\mathrm{UI}(\mu \mathrm{g} / \mathrm{g})$} & \\
\cline { 2 - 3 } Variables & Coefficients & SE & $P$ \\
\hline Intercept & 1.816 & 0.091 & $<0.001$ \\
lodine content of salt (mg/kg) & 0.009 & 0.001 & $<0.001$ \\
Age (years) & 0.007 & 0.003 & $<0.001$ \\
Occupation (\%) & & & $<0.05$ \\
$\quad$ White collar & Reference & & \\
$\quad$ Blue collar & 0.101 & 0.041 & \\
$\quad$ Housewife & 0.019 & 0.026 & \\
$\quad$ Other & 0.024 & 0.019 & \\
Multivitamin supplement & & & $<0.01$ \\
$\quad$ with iodine (15 $\mu \mathrm{g} / \mathrm{d})$ & & & \\
$\quad$ No & Reference & & \\
$\quad$ Yes & 0.089 & 0.032 & \\
Seaweed & & & $<0.001$ \\
$\quad$ No & Reference & & \\
Yes & 0.192 & 0.024 & \\
\hline
\end{tabular}

* Other variables included in the linear models were as follows: educational level; husband's educational level; household income; cows' milk; yogurt; soya sauce; egg; river fish; sea fish; clams; medical history of thyroid; smoking status.

hormones in the second trimester of pregnant women in Shanghai is presented in Table 4. Finally, the correlation between UI and iodine content of salt was analysed using Pearson's test (Fig. 1). A $P$ value $<0.05$ was considered statistically significant. All statistical analyses were performed using Empower(R) (www.empowerstats.com; X\&Y Solutions, Inc.) and R (http://www.R-project.org).

\section{Results}

The median concentrations of UI were $156 \cdot 3,176.9$ and $175 \cdot 1 \mu \mathrm{g} / \mathrm{g}$ creatinine in the first, second and third trimesters of pregnancy, respectively, as shown in Table 1. Accordingly, the prevalence of UI deficiency in the first trimester was $48.3 \%$, which is higher than that in the other two stages (34.2\% in the second trimester and 36.2\% in the third trimester of pregnancy; $P<0 \cdot 05$ ).

Tables 2 and 3 show unadjusted and adjusted influencing factors of UI, respectively. These risk factors were categorised into socio-economic status, sources of dietary iodine in the $24 \mathrm{~h}$ recall, medical history of thyroid and smoking status. Using the generalised linear analysis, the following variables were found to be significant in the model: iodine content of salt $(\beta=0.009, \quad P<0.001)$; age $(\beta=0.007, \quad P<0.001)$; occupation $(P<0.05)$; multivitamin supplement with iodine $(P<0 \cdot 01)$; seaweed intakes $(P<0.001)$ (Table 3$)$. In addition, there was a linear correlation between iodine content of salt and UI $(r$ 0.406, $P<0 \cdot 001$; Fig. 1$)$.

The proportions of normal T3, T4, FT3, FT4 and TSH were $94.0,97,58.2,71.6$ and $78.4 \%$, respectively, in the second trimester of pregnancy, as shown in Table 4.

Table 5 shows the association between UI and thyroid hormone levels in the second trimester of pregnancy. The association between T3, T4, FT3, FT4, TSH and UI was not found to be significant $(P>0 \cdot 05)$.

\section{Discussion}

UI is a good biochemical marker for the evaluation of iodine intake and iodine nutrition status. The WHO has recommended the median UI concentration as the indicator of iodine nutrition status in the general population. The recommended median UI concentration for pregnant women is $150-249 \mathrm{~g} / \mathrm{l}^{(18)}$. In the present study, the median UI concentration in all the three trimesters of pregnancy was found to be within the reference range, but at the low end. However, the prevalence of UI deficiency was high in all the three trimesters of pregnancy. Moreover, the proportion found in the present result was lower than that reported by Zou et al. ${ }^{(9)}$ showing that the prevalence of UI deficiency was $55.4 \%$ for pregnant women in Shanghai.

It is noteworthy that the prevalence of UI deficiency in the first trimester of pregnancy was higher than that in the other trimesters of pregnancy in Shanghai. It is well known that thyroid hormone plays an important role during early brain development in the fetus, whereas fetal thyroid cannot produce thyroid hormones. Normal brain development of the fetus is thus dependent on maternal thyroid hormone via placental transfer in the early stage of pregnancy ${ }^{(19)}$. Iodine is very important for the proper functioning of the thyroid gland. Therefore, iodine deficiency would be considered as one of the influencing factors that interfere with brain development in the fetus during early pregnancy. The high prevalence of UI deficiency in the first trimester of pregnancy may be associated with excess iodine intake resulting in increased thyroid hormone production. (1) Maternal thyroid hormone production during the first trimester of pregnancy obviously increases immediately after its onset, in order to ensure the early surge in circulating FT4, considering, among other factors, that the plasma volume increases rapidly ${ }^{(20)}$. (2) A transient stimulation of the maternal thyroid gland by elevated levels of human chorionic gonadotropin leads to a rise in thyroid hormone levels during the first trimester of pregnancy ${ }^{(21)}$. (3) In Shanghai, some pregnant women began to take multivitamin supplements after their first visit to obstetricians at about 12 weeks of gestation. (4) A lack of the collection of urine samples from the same women may be

Table 4. Prevalence of normal thyroid hormones in the second trimester of pregnancy

(Number of women and percentages, $n$ 134)

\begin{tabular}{|c|c|c|}
\hline Thyroid hormone categories & $n$ & $\%$ \\
\hline \multicolumn{3}{|l|}{ T3 } \\
\hline \multicolumn{3}{|l|}{ T4 } \\
\hline Normal level $(88 \cdot 6-213 \cdot 2 \mathrm{nmol} / \mathrm{l})$ & 130 & 97 \\
\hline \multicolumn{3}{|l|}{ FT3 } \\
\hline $\begin{array}{l}\text { Normal level }(3.55-5.25 \mathrm{pmol} / \mathrm{l}) \\
\text { FT4 }\end{array}$ & 78 & $58 \cdot 2$ \\
\hline Normal level $(10.6-17.6 \mathrm{pmol} / \mathrm{l})$ & 96 & $71 \cdot 6$ \\
\hline \multicolumn{3}{|l|}{ TSH } \\
\hline Normal level $(0.05-4.50 \mathrm{mlU} / \mathrm{l})$ & 105 & 78.4 \\
\hline
\end{tabular}

T3, tri-iodothyronine; T4, thyroxin; FT3, free tri-iodothyronine; FT4, free thyroxine; $\mathrm{TSH}$, thyroid-stimulating hormone. 
a possible explanation for the higher prevalence of iodine deficiency in the first trimester of pregnancy.

In the present study, intake of iodised salt, seaweed and iodine supplement was found to be the main sources of iodine. The present study showed that Shanghai, despite being a coastal city, has low iodine content in natural dietary sources. A recent survey in China has demonstrated that although iodised salt was used in some food processing industries, it was not used in household foods, such as soya source, bread, noodles and mineral water. Seaweed is the only seafood that was positively correlated with UI. Similarly, a Korean study has also found a significant correlation between dietary iodine intake primarily from seaweed ${ }^{(22)}$ and UI excretion. However, seaweed was not common in the daily diet in Shanghai, and only $15 \%$ of the participants in the present survey consumed seaweed intake. Notably, iodised salt is a main source of iodine, and there is a significant correlation between iodine content of salt and UI. In China, salt production is tightly controlled and the sale of non-iodised salt is restricted. Recently, some coastal cities have been unofficially allowing the sale of non-iodised salt, and there were calls for liberalising provincial control of such sales ${ }^{(23)}$. According to official reports from Shanghai, the iodine content in iodised salt available in stores ranges from 20 to 50 parts per million. Non-iodised salt is widely used in the food processing industry. In the present study, $14.9 \%$ of pregnant women did not use iodised salt. This might be a threat to universal salt iodisation policy in China, which could raise the possibility of recrudescence of iodinedeficiency disorders, especially in pregnant women. In addition, supplementation of iodine-rich nutrients during pregnancy, especially in early pregnancy, is needed in order to improve the iodine status of pregnant women.

The present study did not find any correlation between T3, T4, FT3, FT4, TSH and UI in the analysis of a small size sample. Although the representativeness of the samples should be considered, the present result is consistent with that of most other studies ${ }^{(24-28)}$. A recent report from Japan showed no direct correlation between UI and serum TSH or FT4 concentrations during pregnancy ${ }^{(24)}$, nor did a study in

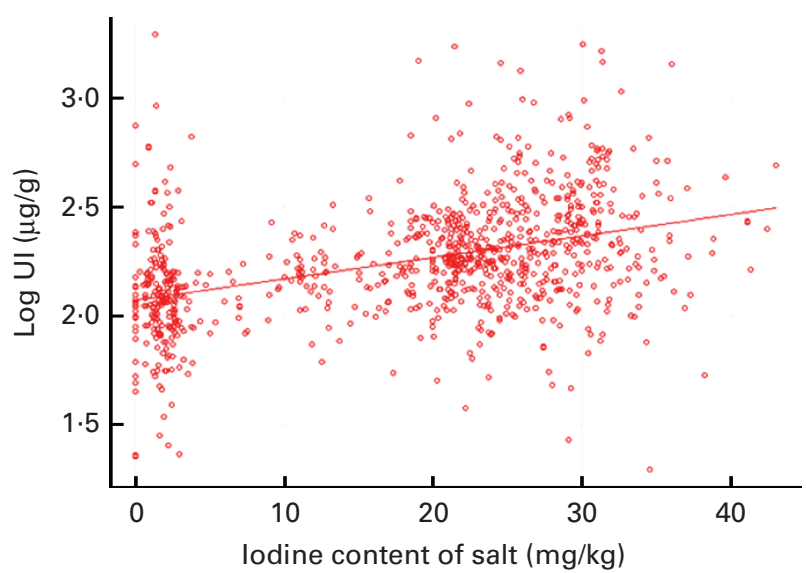

Fig. 1. Correlation between iodine content of salt and urinary iodine (Ul: $r 0.406, P<0.001$ ). A colour version of this figure can be found online at http://www.journals.cambridge.org/bjn
Table 5. Association between urinary iodine (UI) and thyroid hormone levels in the second trimester of pregnancy $(n 134)$

\begin{tabular}{lccccc}
\hline & \multicolumn{5}{c}{ Correlation } \\
\cline { 2 - 6 } & $\begin{array}{c}\text { T3 } \\
(\mathrm{nmol} / \mathrm{l})\end{array}$ & $\begin{array}{c}\mathrm{T} 4 \\
(\mathrm{nmol} / \mathrm{l})\end{array}$ & $\begin{array}{c}\mathrm{FT3} \\
(\mathrm{pmol} / \mathrm{l})\end{array}$ & $\begin{array}{c}\mathrm{FT} 4 \\
(\mathrm{pmol} / \mathrm{l})\end{array}$ & $\begin{array}{c}\mathrm{TSH} \\
(\mu \mathrm{lU} / \mathrm{ml})\end{array}$ \\
\hline Log UI $(\mu \mathrm{g} / \mathrm{g})$ & 0.053 & 0.073 & -0.010 & -0.035 & 0.026 \\
$P$ & $>0.05$ & $>0.05$ & $>0.05$ & $>0.05$ & $>0.05$ \\
\hline
\end{tabular}

T3, tri-iodothyronine; T4, thyroxin; FT3, free tri-iodothyronine; FT4, free thyroxine; $\mathrm{TSH}$, thyroid-stimulating hormone.

northern Spain ${ }^{(25)}$. It has been speculated that the lack of such correlation could be attributed to the amount of iodine reserve in the thyroid gland in a population that do not have persistent iodine deficiency. Thus, thyroid hormone synthesis could be guaranteed ${ }^{(25)}$.

\section{Limitations}

The present study has some limitations. First, a previous history of chronic thyroiditis and subclinical hypothyroidism cannot be ruled out based on patient's anamnesis in their subclinical course. Serum anti-thyroid peroxidase antibodies, an independent and important predictor of subclinical hypothyroidism and chronic thyroiditis, were not measured in the present study. Second, we neither tested hormone levels nor addressed the association between thyroid hormone levels and UI during pregnancy, especially in the first trimester of pregnancy. Further research is needed to clarify whether UI would be associated with thyroid hormone concentration in the first trimester of pregnancy.

\section{Conclusion}

In summary, there is a high prevalence of UI deficiency in pregnant women in Shanghai, especially during the first trimester of pregnancy. Both iodine content of household salt and multivitamin supplement with iodine are the main determinants of UI levels.

Nutritional status of iodine in pregnant women is not well recognised in Shanghai. Thus, it is essential to publicise the knowledge of iodine-deficiency disorders, and its potential risk for fetal neurodevelopment. Regular monitoring of iodine nutrition during pregnancy needs to be considered seriously by health authorities, policy makers and relevant organisations. The use of iodised salt and supplementation of iodine-rich nutrients during pregnancy are recommended.

\section{Acknowledgements}

The present study was supported by the Minhang District Maternal and Child Health Hospital, Shanghai, China. The authors are grateful to the researchers X. Z. and L. J. who were responsible for the collection of the sample and data.

The present study was funded by the National Natural Science Foundation, China (grant no. 81373004) and the Establishing a Platform for Clinical Research Data Sharing to 
Facilitate Multi-Center Collaboration by China-Canada Joint Effort (2014DFG31460).

The authors' contributions are as follows: X. Y. designed the analyses of the study and performed the statistical analyses; Z. W. and W. W. were involved in the writing of the manuscript; J. Z. contributed to the statistical counselling; X. Y., Z. W. and W. W. participated in the discussion of the result and revision of the manuscript; X. Z. and L. J. were responsible for the collection of the sample and data.

There are no conflicts of interest.

\section{References}

1. Caldwell KL, Jones R \& Hollowell JG (2005) Urinary iodine concentration: United States National Health and Nutrition Examination Survey 2001-2002. Thyroid 15, 692-699.

2. Pearce EN (2013) Monitoring and effects of iodine deficiency in pregnancy: still an unsolved problem? Eur J Clin Nutr 67, 481-484

3. Hetzel BS (2000) Iodine and neuropsychological development. J Nutr 130, 493S-495S.

4. Mitka M (2013) Even mild iodine deficiency during gestation may impair brain function in children. JAMA 309, $2428-2428$.

5. Skeaff SA (2012) Assessing iodine intakes in pregnancy and strategies for improvement. J Trace Elem Med Biol 26, $141-144$

6. Hollowell JG, Staehling NW, Hannon WH, et al. (1998) Iodine nutrition in the United States. Trends and public health implications: iodine excretion data from National Health and Nutrition Examination Surveys I and III (1971-1974 and 1988-1994). J Clin Endocrinol Metab 83, 3401-3408.

7. Nicola JP, Basquin C, Portulano C, et al. (2009) The $\mathrm{Na}^{+} / \mathrm{I}^{-}$ symporter mediates active iodide uptake in the intestine. Am J Physiol Cell Physiol 296, C654-C662.

8. Kusić Z, Jukić T, Rogan SA, et al. (2012) Current status of iodine intake in Croatia - the results of 2009 survey. Coll Antropol 36, 123-128.

9. Zou S, Wu F, Guo C, et al. (2012) Iodine nutrition and the prevalence of thyroid disease after salt iodization: a crosssectional survey in Shanghai, a coastal area in China. PLOS ONE 7, e40718.

10. Dunn JT, Crutchfield HE, Gutekunst R, et al. (1993) Two simple methods for measuring iodine in urine. Thyroid $\mathbf{3}$, 119-123.

11. Caldwell KL, Makhmudov A, Ely E, et al. (2011) Iodine status of the US population, National Health and Nutrition Examination Survey, 2005-2006 and 2007-2008. Thyroid 21, 419-427.

12. Zimmermann MB, Zeder C, Chaouki N, et al. (2003) Dual fortification of salt with iodine and microencapsulated iron: a randomized, double-blind, controlled trial in Moroccan schoolchildren. Am J Clin Nutr 77, 425-432.
13. Pino S, Fang SL \& Braverman LE (1996) Ammonium persulfate: a safe alternative oxidizing reagent for measuring urinary iodine. Clin Chem 42, 239-243.

14. Burns R, Mayne PD, O'Herlihy C, et al. (2008) Can neonatal TSH screening reflect trends in population iodine intake? Thyroid 18, 883-888.

15. Anckaert E, Poppe K, Van Uytfanghe K, et al. (2010) FT4 immunoassays may display a pattern during pregnancy similar to the equilibrium dialysis ID-LC/tandem MS candidate reference measurement procedure in spite of susceptibility towards binding protein alterations. Clin Chim Acta 411, $1348-1353$.

16. Demers LM \& Spencer CA (2003) Laboratory medicine practice guidelines: laboratory support for the diagnosis and monitoring of thyroid disease. 58, 138-140.

17. Yan YQ, Dong ZL, Dong L, et al. (2011) Trimester- and method-specific reference intervals for thyroid tests in pregnant Chinese women: methodology, euthyroid definition and iodine status can influence the setting of reference intervals. Clin Endocrinol 74, 262-269.

18. WHO, ICCIDD \& UNICEF (2007) Assessment of the Iodine Deficiency Disorders and Monitoring their Elimination, 3rd ed. Geneva: WHO.

19. Toft A (2004) Increased levothyroxine requirements in pregnancy - why, when, and how much. $N$ Engl J Med 351, 292-294.

20. de Escobar GM, Obregon MJ \& Del Rey FE (2004) Role of thyroid hormone during early brain development. Eur $J$ Endocrinol 151, Suppl. 3, U25-U37.

21. Glinoer D (2001) Pregnancy and iodine. Thyroid 11, 471-481.

22. Kim JY, Moon SJ, Kim KR, et al. (2000) Dietary iodine intake and urinary iodine excretion in normal Korean adults. Yonsei Med J 39, 355-362.

23. Wu Y, Li X, Chang S, et al. (2012) Variable iodine intake persists in the context of universal salt iodization in China. J Nutr 142, 1728-1734.

24. Fuse Y, Ohashi T, Yamaguchi S, et al. (2011) Iodine status of pregnant and postpartum Japanese women: effect of iodine intake on maternal and neonatal thyroid function in an iodine-sufficient area. J Clin Endocrinol Metab 96, 3846-3854.

25. Aguayo A, Grau G, Vela A, et al. (2013) Urinary iodine and thyroid function in a population of healthy pregnant women in the North of Spain. J Trace Elem Med Biol 27, 302-306.

26. Yarrington CD \& Pearce EN (2011) Dietary iodine in pregnancy and postpartum. Clin Obstet Gynecol 54, 459-470.

27. Glinoer D, De Nayer P, Bourdoux P, et al. (1990) Regulation of maternal thyroid during pregnancy. J Clin Endocrinol Metab 71, 276-287.

28. Luton D, Alberti C, Vuillard E, et al. (2011) Iodine deficiency in northern Paris area: impact on fetal thyroid mensuration. PLOS ONE 6, e14707. 